\title{
Online Transdiagnostic Treatment for Emotional Disorders
}

\section{B A C K G R O U N D}

Worldwide, mental disorders are a serious public health problem with a high social cost that affects people without distinction of age, sex, socioeconomic status or culture. In Mexico, the last National Survey of Psychiatric Epidemiology (2003) reported that among the most frequent disorders that the population presents are those related to anxiety $(14.3 \%)$; while depression represents $4.3 \%$ of the global burden of mental disorders $[1,2,3]$

From the dimensional perspective, the consideration of the causes and coping resources which the person counts and not only the counting of prescribed grouped symptoms takes relevance. Thus, transdiagnostic treatments could help overcome the inconveniences related to comorbidity between disorders and thereby open the way to a new generation of treatments focused on emotion [4].

The Unified Protocol for Transdiagnostic Treatment of Emotional Disorders [UPTTED] addresses four main components to reduce emotional dysregulation: emotional avoidance, promotion of cognitive flexibility, exposure to situations and feelings avoided, and emotional awareness centered on the present.

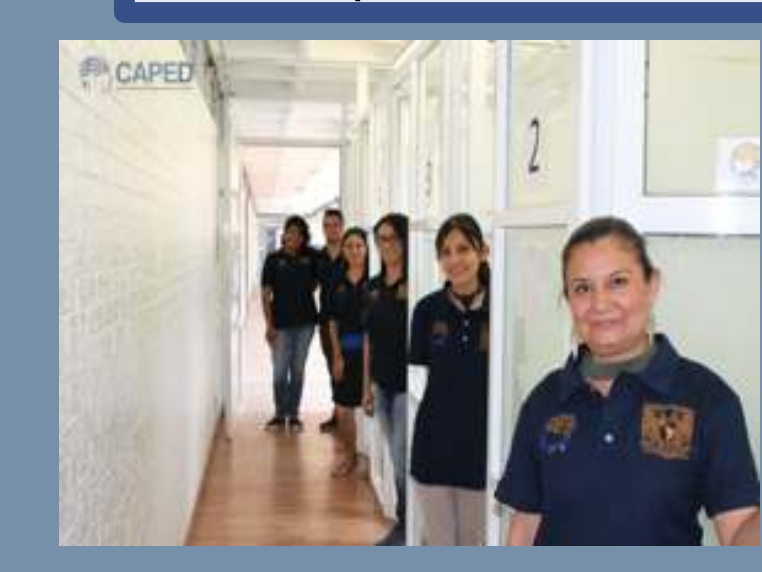

CAPED Advisor in session

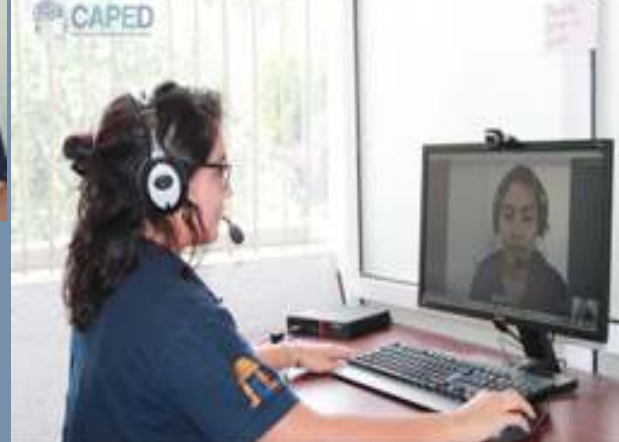

\section{METHOD}

PARTICIPANTS: 18 female students participated in the

online Psychology course, which requested the psychological assistance service to the Psychological and Educational Distance Support Center and referred situations of emotional distress (depression and/or anxiety). The criteria of inclusion was: (a) be of legal age (>18); (b) voluntarily participate in the study; (c) meet diagnostic criteria for emotional disorders (anxiety or depression) and show a score $\leq 25$ on the Beck Anxiety Depression (BAI) [5] and/or $\leq 30$ on the Depression Inventory of Beck (BDI) [6] (d) have access to a computer with Internet connection, (e) a valid email address. The participants were randomly divided to each condition ( $n=9$ transdiagnostic treatment and $n=9$ control group).

DESIGN

An experimental design was used between subjects with two independent groups, with intrasubject measures in two moments of evaluation: pretest and posttest [49]. The participants were randomly assigned to two conditions: (a) online transdiagnostic intervention and (b) online cognitive-behavioral intervention.

\section{INSTRUMENTS}

- Beck Depression Inventory (BDI). Standardized in Mexico by Jury, Villegas, Méndez, Rodríguez, Loperena and Varela (1998) [5].

-Beck Anxiety Inventory (BAI). Spanish version by Robles, Varela, Jurado and Páez (2001) [6] -Emotion Regulation Scale (DERS). Spanish version by Muñoz-Martínez, Vargas and Hoyos-González (2016) [7]

- Training manual for online counseling [8]. Realized with the objective of providing basic for psychological counseling.

-Intelligent Evaluation System and Interface for video conferencing and Chat on Moodle platform.

MATERIALS

Training manual for online counseling [8]. Realized with the objective of providing basic for psychological raining to direct an intervention of emotional online counseling.

-Intelligent Evaluation System and Interface for video conferencing and Chat on Moodle platform.

\section{RE S U L T S}

The results presented by each group show that the treatment had the expected effect by reducing statistically significant scores in the three variables evaluated in both intervention groups, in the experimental condition (UP): BDI $(z=0.35, p=0.05, d=-1.89)$, BAI $(z=0.38$, $\mathrm{p}=0.05, \mathrm{~d}=-2.07)$ and DERS $(\mathrm{z}=0.00, \mathrm{p}=0.05$, $\mathrm{d}=-2.11)$; in the CBT condition the results were: $\mathrm{BDI}(\mathrm{z}=0.35, \mathrm{p}=0.05, \mathrm{~d}=-2.10)$, BAl $(z=0.15, p=0.05, d=-2.43)$, DERS $(z=0.008$ $p=0.05, d=-2.66$ )

No significant differences were found between the groups $(U=0.605, p=0.05)$, in the BAI $(U=0.436, p=0.05)$ and in the DERS $(\mathrm{U}=0.931, \mathrm{p}=0.05)$.

Table 1. Means of scores obtained before and after treatment

\begin{tabular}{|llllr|}
\hline \multicolumn{2}{|c|}{ UP } & \multicolumn{2}{c|}{ CBT } \\
\hline Measure & $\begin{array}{c}\text { Pretest } \\
\text { M(S.D) }\end{array}$ & $\begin{array}{c}\text { Postest } \\
\text { M(S.D) }\end{array}$ & $\begin{array}{c}\text { Pretest } \\
\text { M(S.D) }\end{array}$ & $\begin{array}{r}\text { Postest } \\
\text { M(S.D) }\end{array}$ \\
\hline BDI & $14.4(6.4)$ & $7.3(3)$ & $18.8(14.8)$ & $8.3(8.4)$ \\
\hline BAI & $21.5(14.6)$ & $7.2(3.9)$ & $18.5(15.5)$ & $5.3(3.2)$ \\
\hline DERS & $25.8(12.3)$ & $12.2(7.8)$ & $29.8(16.2)$ & $12(6.2)$ \\
\hline $\begin{array}{l}\text { Note: UP= Unified Protocol Group; CBT= Cognitive Behavior Treatment } \\
\text { Group; M= media, S. D. = Standard Deviation; BDI= Beck Depression } \\
\text { Inventory; BAI= Beck Anxiety Inventory; DERS=Difficulties in Emotion } \\
\text { Regulation Scale. }\end{array}$ \\
\hline
\end{tabular}

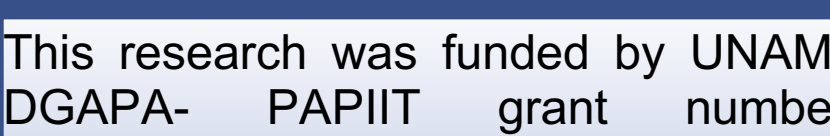

C O N T A T TA300219.

口都口

\section{DISCUSSION}

The main purpose of the present study was to test a transdiagnostic intervention for emotional disorders via Internet. The results obtained show the effectiveness of the treatment based on the unified protocol for cases with different manifestations of emotions of anxiety and depression in a sample of Mexican participants. No differences were found between groups when evaluating the efficacy of the treatments, but the most relevant data was that in both conditions there was a statistically significant decrease in relation to the pretest. In this sense, the clinical gains in the participant are extrapolated to other areas of the user's life, Reyes (2013) [9] mentions that the transdiagnostic model has results reflected in the emotional regulation while decreasing the present symptomatology.

This work has limitations that it is important to point out, the use of self-reports, since it does not allow to directly verify the reduction of symptoms of anxiety or depression and with it, to have the threat of social desirability of the participant [10].

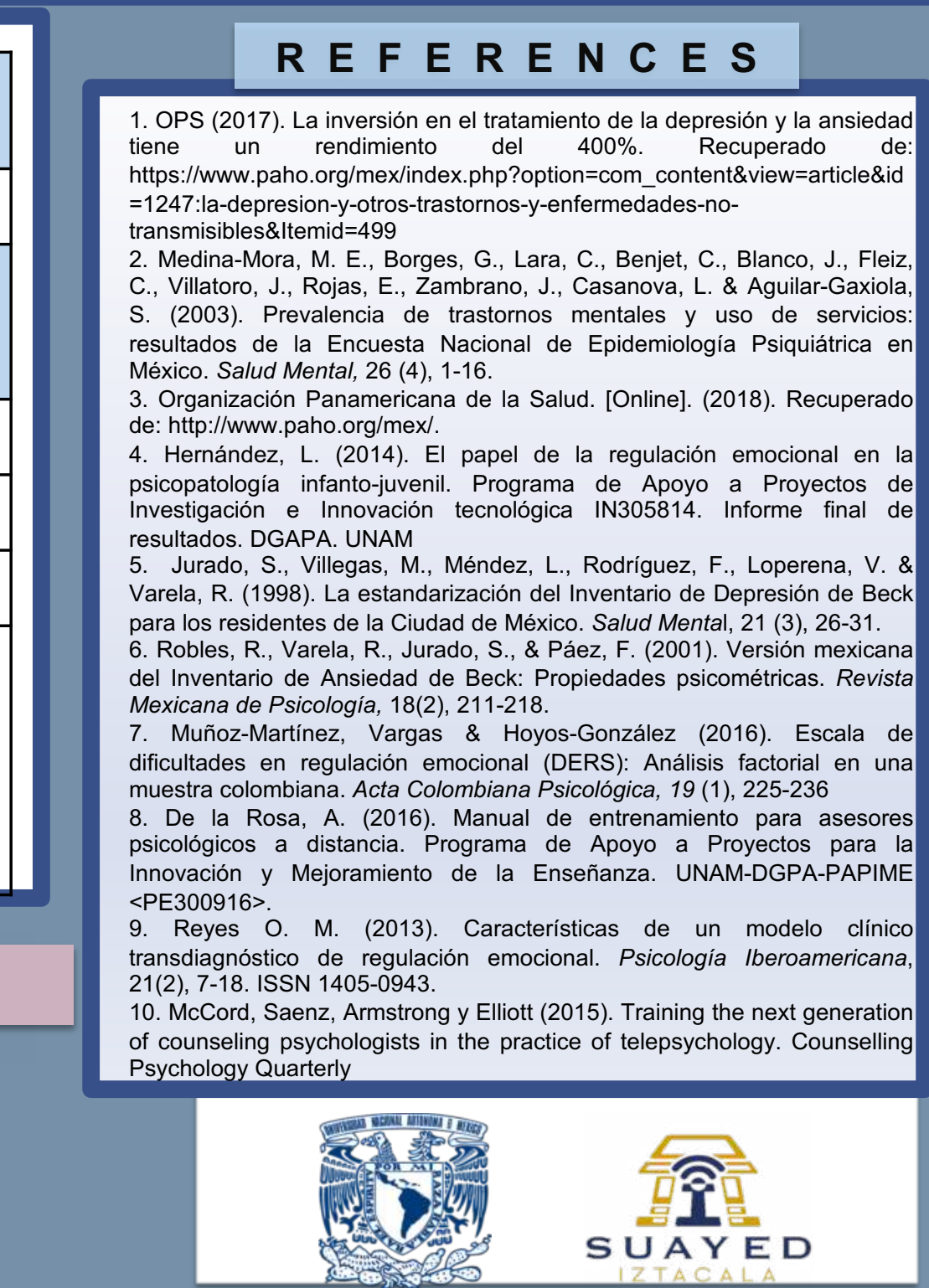

